\title{
Asymptotic analysis of a ferromagnetic Ising system with "diffuse" interfacial energy
}

\author{
Andrea Braides* \\ Dipartimento di Matematica, Università di Roma Tor Vergata \\ via della ricerca scientifica 1, 00133 Roma, Italy \\ Andrea Causin and Margherita Solci \\ DADU, Università di Sassari \\ piazza Duomo 6, 07041 Alghero (SS), Italy
}

\begin{abstract}
We give an example of a one-dimensional scalar Ising-type energy with longrange interactions not satisfying standard decay conditions and which admits a continuum approximation finite for all functions $u$ in $B V((0, L),[-1,1])$ and taking into account the total variation of $u$. The optimal discrete arrangements show a periodic pattern of interfaces. In this sense, the continuum energy is generated by "diffuse" microscopic interfacial energy. We also show that related minimum problems show boundary and size effects in dependence of $L$.
\end{abstract}

Keywords: Ising-type energies, discrete-to-continuum, interfacial energies, size effects MSC Classification (2010): 49J45, 49Q20, 82B20

\section{Introduction}

Despite the vast Statistical Mechanics literature on Ising-type systems, in particular those parameterized on a lattice, their treatment from the variational standpoint is relatively recent and still incomplete. In the simplest case, such systems can be seen as driven by an energy

$$
-\sum_{i, j} c_{i j} u_{i} u_{j}
$$

where $i, j$ belong to some subset of a lattice $\mathcal{L}$ and the variable $u_{i}$ takes the value in $\{-1,+1\}$. Note that in the ferromagnetic case (i.e., when $c_{i j} \geq 0$ for all $i, j$ ), up to

\footnotetext{
*Corresponding author: e-mail braides@mat.uniroma2.it, tel.(+39)0672594688
} 
additive and multiplicative constants, it is more handy to rewrite the energies in (1) as

$$
\sum_{i, j} c_{i j}\left(u_{i}-u_{j}\right)^{2}
$$

in order to have minimizers with zero energy and to avoid $+\infty-\infty$ indeterminate forms in the case of infinite domains. The paper [14] by Caffarelli and de la Llave provided a first homogenization result for periodic ferromagnetic Ising-type systems by characterizing ground states as plane-like minimizers (see also the recent paper [6]).

A later work by Alicandro et al. [1] formalized the treatment of such systems in terms of $\Gamma$-convergence. Those authors set the problem in the framework of a discrete-tocontinuum analysis, scaling the energies and characterizing the continuum limit within the theory of interfacial energies defined on partitions by Ambrosio and Braides [4], and also treating some antiferromagnetic case. In that approach the energies are scaled by a small parameter $\varepsilon>0$ as

$$
\sum_{i, j} \varepsilon^{d-1} c_{i j}\left(u_{i}-u_{j}\right)^{2}
$$

where now $i, j$ are supposed to belong to $\varepsilon \mathcal{L}$ and $d$ is the dimension of the ambient space; i.e., $\mathcal{L} \subset \mathbb{R}^{d}$. Note the $d-1$-th power in (3), corresponding to a surface scaling.

In the case of ferromagnetic systems, the macroscopic magnetization parameter $u$ still only takes the values \pm 1 , and the continuum-limit energy has the form

$$
\int_{\partial\{u=1\}} \varphi(\nu) d \mathcal{H}^{d-1},
$$

where $\nu$ is the measure-theoretical normal to the set of finite perimeter $\{u=1\}$. A general homogenization theorem within the class of ferromagnetic spin system was proved by Braides and Piatnitski also allowing for random coefficients [11]. In all those cases the limit problem is of the form (4). Applications of this result comprise the description of quasicrystalline structures [7, 12] and optimal design problems for networks [9, 10]. Moreover, the homogenization result for periodic systems has been recently extended to some types of antiferromagnetic interactions when the limit is instead parameterized on partitions into sets of finite perimeter [8] and can be written as a sum of energies of the form (4).

Alicandro and Gelli [3] have recently remarked that if we take $\varepsilon$-depending coefficients, i.e., we allow for energies of the form

$$
\sum_{i, j} c_{i j}^{\varepsilon}\left(u_{i}-u_{j}\right)^{2}
$$

in place of (2), still within the framework of the discrete-to continuum analysis of ferromagnetic systems with surface scaling, then the limit functional might contain 
non-local terms, of the form

$$
\int k(x, y, u(x)-u(y)) d \mu(x, y)
$$

for suitable measures $\mu$ and kernels $k$.

Different scalings of the energies are also possible. In [2] Alicandro et al. have examined the bulk scaling

$$
\sum_{i, j} \varepsilon^{d} f\left(u_{i}, u_{j}\right)
$$

for general $f$ (and $u_{i}$ taking values in a more general set), showing that the limit is a bulk integral

$$
\int g^{* *}(u) d x
$$

$\left(g^{* *}\right.$ denotes the convex envelope of $g$ ). Note however that in the ferromagnetic spin case $g$ is trivial, and can be interpreted as a double-well potential with minima in \pm 1 . As a consequence, when the hypothesis of [3] are satisfied, formally, ferromagnetic Ising-type systems can be approximated in the continuum as an expansion

$$
\int g(u) d x+\varepsilon \int_{\partial\{u=1\}} \varphi(\nu) d \mathcal{H}^{d-1}+\ldots
$$

(see $[5,13])$. This expression highlights a separation of scales effect, which suggests that either a bulk or a surface scaling have to be taken into account (depending on the problem at hand), unless higher-order scalings come into play.

In this paper we show that this is not the case for general $\varepsilon$-depending Ising-type energies with a simple example in dimension one. Even though we maintain the suggestive terminology of Statistical Mechanics, our motivation is towards the understanding of the behaviour of general lattice systems with a complex topology of the interactions beyond the application to problems in Physics. To that end, the choice of the simplest type of interactions (ferromagnetic) allows to focus our analysis on the effect of the topology of the graph of the interacting sites of the lattice.

In the notation above, the energies we will examine can be written as in (5) with $c_{i j}^{\varepsilon}=1$ if $|i-j|=1$ or $|i-j|=\lfloor 1 / \sqrt{\varepsilon}\rfloor$, and $c_{i j}^{\varepsilon}=0$ otherwise. These energies have long-range interactions which do not satisfy the decay conditions on $c_{i j}^{\varepsilon}$ required in [3] to obtain an integral representation as an interfacial energy. The key feature of these energies is the non-trivial topology of the corresponding graph, which corresponds to that of a two-dimensional square lattice with nearest-neighbour interactions, and not the range of the interaction themselves. Indeed, note that if we take into account all interactions up to distance $\lfloor 1 / \sqrt{\varepsilon}\rfloor$, e.g., taking $c_{i j}^{\varepsilon}=1$ if $|i-j| \leq\lfloor 1 / \sqrt{\varepsilon}\rfloor$ and $c_{i j}^{\varepsilon}=0$ otherwise, this can instead be seen as a superposition of usual one-dimensional nearestneighbour ferromagnetic lattice Ising systems and, after the proper renormalization, 
gives a sharp-interface energy; i.e., with the constraint on the limit energy domain that $u \in\{-1,1\}$ almost everywhere (see Remark 3 ).

We will instead show that energies (5) have a meaningful limit, which is not of the forms described above, at an intermediate scale between the bulk and surface scales (namely, at the scale $\sqrt{\varepsilon}$ ). More precisely, if we examine the discrete-to-continuum limit of

$$
\sum_{i, j} \sqrt{\varepsilon} c_{i j}^{\varepsilon}\left(u_{i}-u_{j}\right)^{2}
$$

restricted to the portion of $\varepsilon \mathbb{Z}$ contained in an interval $(a, b)$, then the continuum parameter $u$ is a function with bounded variation taking values in $[-1,1]$, and, denoted by $D u$ its derivative in the sense of distributions (which is a measure on $(a, b)$ ), we have a limit energy

$$
2|\{x \in(a, b):-1<u(x)<1\}|+|D u|(a, b) .
$$

Hence, the effect of the interaction coefficients is not strong enough to force that $u \in$ $\{ \pm 1\}$ but strong enough to give a dependence on $|D u|$.

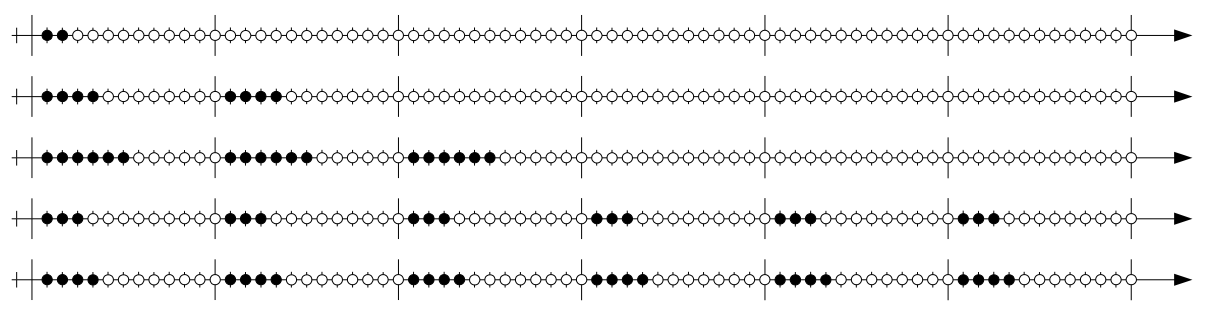

Figure 1: Pictorial representation of optimal configurations for five different volume fractions: each line represents the "shape" of a minimizer of the limit energy with a prescribed integral; the black dots stand for $u=1$

Contrary to the usual sharp-interface limit models, where the corresponding discrete functions simply exhibit a sharp separation of the indices where the value is 1 or -1 , optimal arrangements for the energy in (11) highlight interesting features of the optimal arrangements for the discrete energy. Such geometries show a periodic pattern of the interfaces. In this sense, we interpret the limit energy as generated by a "diffuse" microscopic interfacial energy. In order to highlight the effect of these microgeometries, the behaviour of some minimum problems will be studied in Section 4. In Fig. 1 we picture discrete minimizers $u$ of the limit energy in a small (fixed) interval $(a, b)$, under the constraint of prescribed average $|b-a|^{-1} \int_{a}^{b} u d t=\sigma$. In the terminology of Statistical Physics those minimizers would describe microscopic states for a value $\sigma$ of the limit magnetization. We represent five different cases with increasing values of the integral average $\sigma \in[-1,0]$, the case $\sigma \in[0,1]$ being symmetric. Circles represent points 
in $\varepsilon \mathbb{Z}$, the black ones being those where $u_{i}=1$ and the white ones those where $u_{i}=-1$. For values of $\sigma$ close to -1 we have a partial concentration on one side of the interval, which grows until a certain threshold, after which the function $u$ is (approximately) periodically distributed over the whole interval. We note that this description is much easier when obtained from the continuum energy rather than directly examining the discrete energies.

The idea behind the proof of the continuum approximation is that the energy in (5) can be equivalently interpreted as defined on the two-dimensional lattice $\lfloor 1 / \sqrt{\varepsilon}\rfloor \mathbb{Z}^{2}$ if nearest-neighbours in $\mathbb{Z}$ are interpreted as nearest-neighbours in the vertical direction in $\lfloor 1 / \sqrt{\varepsilon}\rfloor \mathbb{Z}^{2}$ and, correspondingly, $\lfloor 1 / \sqrt{\varepsilon}\rfloor$-neighbours in $\mathbb{Z}$ as nearest-neighbours in the horizontal direction in $\lfloor 1 / \sqrt{\varepsilon}\rfloor \mathbb{Z}^{2}$. With this identification the energy becomes a simple nearest-neighbour interaction energy in dimension two, of which we can compute the $\Gamma$-limit in the surface scaling. Reinterpreting the limit in dimension one gives the form (11) after some technical arguments.

The interest in this example is that the limit is characterized by the non-trivial topology of the graph of the connections $i, j$ with $c_{i j}^{\varepsilon}=1$. This argument is different from the measure-theoretical ones used in the previous articles cited above, and can be generalized to systems of interactions whose graph of connections can be mapped into an $n$-dimensional Bravais lattice. We hope it will be useful to give a better understanding of the variational approximation of Ising-type systems on general graphs [15].

We complement the analysis with a study of the minimum problems for the limit energy both when a volume constraint is taken into account and when periodic conditions are imposed, thus recovering the behaviour of minimizers for the discrete problems by $\Gamma$-convergence. It is interesting to note how the "two-dimensional" topology of the lattice interactions produces a complex structure of the minimum energy landscape in dependence of the parameters of the problem, and in particular a size effect highlighted by the dependence on the width of the underlying interval. Furthermore, in the periodic case another parameter intervenes given by the "defect" of $\lfloor 1 / \sqrt{\varepsilon}\rfloor$-periodicity of the interval (normalized to a number between 0 and 1). Correspondingly, a boundary term must be added to the $\Gamma$-limit, which further influences the shape of minimizers for certain values of that parameter.

The paper is organized as follows: in Section 2 we state the main $\Gamma$-convergence result (Theorem 1), whose proof is given in Section 3 by showing the lower and upper inequalities. In Section 4 we examine the behaviour of the minimizers of the functionals subjected to a volume constraint; in particular, we prove a compatibility result (Theorem 6) and we describe the "size effect" on the shape of the minimizer in dependence on the size of the domain and on the volume. In Section 5 we conclude the analysis by studying the effect of periodic boundary conditions. The $\Gamma$-convergence result in the periodic case is given in Theorem 10 . 


\section{Statement of the result}

For the sake of notational simplicity, we consider a discrete parameter $n \in \mathbb{N}$ in the place of $\varepsilon$. In the notation used in the Introduction we choose $\varepsilon=\frac{1}{n^{2}}$, so that $\frac{1}{\sqrt{\varepsilon}}=n$. Moreover, we will consider spin functions with values in $\{0,1\}$ instead of $\{-1,1\}$.

For each $n \in \mathbb{N}$ we define

$$
\mathcal{E}_{n}=\left\{\{i, j\}: i, j \in \mathbb{N} \cap\left(0, n^{2}\right] \text { and }|i-j|=1 \text { or }|i-j|=n\right\} .
$$

This set of indices corresponds to $i, j$ such that $c_{i j}^{\varepsilon} \neq 0$ in (10).

Let $\mathcal{A}_{n}$ be the set of the functions $u:(0,1] \rightarrow\{0,1\}$ with $u$ constant on each interval $\left(\frac{i-1}{n^{2}}, \frac{i}{n^{2}}\right], i=1, \ldots, n^{2}$. Such a $u$ corresponds to a discrete function, which we still denote by $u$, defined as its restriction to $\left(\frac{1}{n^{2}} \mathbb{N}\right) \cap(0,1]$. We will denote $u_{i}$ for $u\left(\frac{i}{n^{2}}\right)$.

Now, for $u \in \mathcal{A}_{n}$ we define the functional

$$
F_{n}(u)=\frac{1}{n} \sum_{\{i, j\} \in \mathcal{E}_{n}}\left(u_{i}-u_{j}\right)^{2} .
$$

This is a rewriting of energy (10), with the scaling $\frac{1}{n}$, and with the constraint $u_{i} \in$ $\{0,1\}$ instead of $u_{i} \in\{-1,+1\}$. Note that this corresponds to a scaling $\sqrt{\varepsilon}$, which is intermediate between the bulk scaling $\varepsilon$ and the surface scaling (since the latter corresponds to no scaling of the energy in dimension one).

We prove the following $\Gamma$-convergence result.

Theorem 1. The sequence of functionals $\left\{F_{n}\right\} \Gamma$-converges with respect to the weak-* convergence in $L^{\infty}(0,1)$ to the functional $F: L^{\infty}(0,1) \rightarrow[0,+\infty]$ given by

$$
F(u)= \begin{cases}2 \mathcal{H}^{1}(\{x: 0<u(x)<1\})+|D u|(0,1) & \text { if } u \in B V(0,1), 0 \leq u \leq 1 \\ +\infty & \text { otherwise }\end{cases}
$$
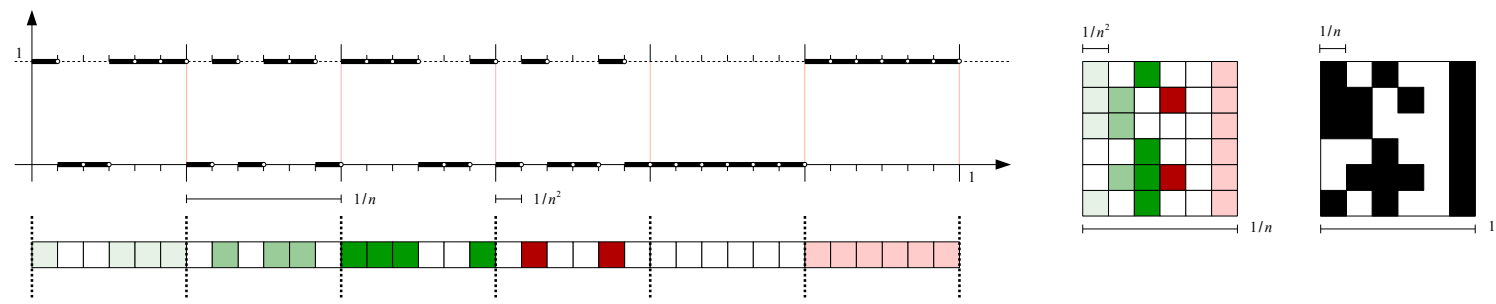

Figure 2: Identification of a spin function with a set in $\mathbb{R}^{2}$ 
To explain the form of the limit energies it is convenient to reinterpret the energies $F_{n}$ in a two-dimensional setting. Indeed, the decomposition $\frac{i}{n^{2}}=\frac{h_{i}-1}{n}+\frac{k_{i}}{n^{2}}$ with $h_{i}, k_{i} \in\{1, \ldots, n\}$ induces a one-to-one correspondence between $\left(\frac{1}{n^{2}} \mathbb{N}\right) \cap(0,1]$ and $\left(\frac{1}{n} \mathbb{N}^{2}\right) \cap(0,1]^{2}$ given by $\frac{i}{n^{2}} \mapsto \frac{1}{n}\left(h_{i}, k_{i}\right)$. With this construction, we map each interval $\left(\frac{i-1}{n^{2}}, \frac{i}{n^{2}}\right]$ to the square $\left(\frac{h_{i}-1}{n}, \frac{h_{i}}{n}\right] \times\left(\frac{k_{i}-1}{n}, \frac{k_{i}}{n}\right]$ so that any function $u \in \mathcal{A}_{n}$ can be represented as the characteristic function of a subset of $(0,1]^{2}$ (see Fig. 2). Hence, we will study the asymptotic behaviour of the sequence $F_{n}$ as $n \rightarrow+\infty$ by using the results for the $\Gamma$-convergence for nearest-neighbour interaction energies in the two-dimensional square lattice $\frac{1}{n} \mathbb{Z}^{2}$. These energies are defined by

$$
E_{n}(v ; \Omega)=\frac{1}{n} \sum_{\left\{z, z^{\prime}\right\} \in \mathcal{N}(n \Omega)}\left(v_{z}-v_{z^{\prime}}\right)^{2}
$$

for $v: \frac{1}{n} \mathbb{Z}^{2} \rightarrow\{0,1\}$ and $\Omega$ a Lipschitz open subset of $\mathbb{R}^{2}$. The sum is running over the set $\mathcal{N}(n \Omega)$ of the pairs of nearest neighbours in $n \Omega \cap \mathbb{Z}^{2}$, and $v_{z}$ stands for $v\left(\frac{z}{n}\right)$. The behaviour of $E_{n}$ is characterized by the following result (see [1, Th. 4.1] and also [14]), where it is understood that each function $v$ is extended as a piecewise-constant function to each square $z+\left[0, \frac{1}{n}\right)^{2}$.

Proposition 2 ([1] Theorem 4.1). The sequence $\left\{E_{n}\right\}$ is equicoercive on $L_{\text {loc }}^{1}(\Omega)$. Its $\Gamma$-limit in the strong $L^{1}(\Omega)$ convergence is finite only on functions $u \in B V(\Omega ;\{0,1\})$; i.e., on characteristic functions of sets of finite perimeter, and its value is

$$
F^{1}(u)=\operatorname{Per}_{1}(\{u=1\} ; \Omega):=\int_{\partial^{*}\{u=1\}}\left\|\nu_{u}\right\|_{1} d \mathcal{H}^{1},
$$

where $\nu_{u}$ denotes the interior normal to $\partial^{*}\{u=1\}$ and $\|\nu\|_{1}=\left|\nu_{1}\right|+\left|\nu_{2}\right|$.

Remark 3. We now remark that considering energies taking into account all interactions up to distance $\lfloor 1 / \sqrt{\varepsilon}\rfloor$ on the same energy scale gives a sharp-interface limit, highlighting that the relevant feature of our result is the non-trivial topology of the graph of the interactions and not the size of their range.

We take $c_{i j}^{\varepsilon}=1$ if $|i-j| \leq\lfloor 1 / \sqrt{\varepsilon}\rfloor$ and $c_{i j}^{\varepsilon}=0$ otherwise. The correct scaling gives the energy

$$
E_{\varepsilon}(u):=\sum_{k=1}^{1 / \sqrt{\varepsilon}} \sum_{i} \varepsilon\left(u_{i+k}-u_{i}\right)^{2}
$$

The $\Gamma$-limit $F$ of such an energy is finite only if $u \in B V((0,1),\{0,1\})$, in which case we have $F(u)=\frac{1}{2} \#(S(u))$, where $S(u)$ is the set of essential discontinuity points of $u$.

The proof of this fact does not follow from the theory in [3], but may be obtained by a coarse-graining argument. We only sketch the proof: take a sequence $\left\{u_{\varepsilon}\right\}$ with 
equibounded energy. With $0<\eta<1 / 8$ fixed, we subdivide the indices into families of $\frac{1}{2 \sqrt{\varepsilon}}$ successive indices (we may suppose that this value is an integer). We first note that $E_{\varepsilon}\left(u_{\varepsilon}\right)$ is larger than a constant (independent of $\eta<1 / 8$ ) times the number of pairs of consecutive families of indices on which the average is larger than $1-\eta$ and lower that $\eta$, respectively. Moveover, $E_{\varepsilon}\left(u_{\varepsilon}\right)$ is also larger than a constant (depending on $\eta$ ) times the number of families for which the average of $u_{\varepsilon}$ on such a family is between $\eta$ and $1-\eta$. Hence there is an equibounded number of such families, independent of $\varepsilon$. We then deduce that we have a finite number of intervals on which either $u \in[1-\eta, 1]$ or $u \in[0, \eta]$. By the arbitrariness of $\eta$ we deduce that $B V((0,1),\{0,1\})$. Once it is proven that the limit $u$ has only a finite number of interfaces, the proof of the $\Gamma$-limit is a simple computation, taking the function $u$ itself (or, more precisely, its discretization) as a recovery sequence. As a result we obtain

$$
\lim _{\varepsilon \rightarrow 0} \varepsilon \sum_{k=1}^{1 / \sqrt{\varepsilon}} k=\frac{1}{2}
$$

as the energy of each interface.

\section{Proof of the result}

We separately prove the upper and lower bounds for the $\Gamma$-limit.

Proposition 4 (Lower bound). Let $\left\{u_{n}\right\}$ be a sequence with $u_{n} \in \mathcal{A}_{n}$ such that $F_{n}\left(u_{n}\right)$ is equibounded. Then there exists $u \in B V(0,1)$ such that, up to subsequences, $u_{n} \stackrel{*}{\rightarrow} u$ in $L^{\infty}(0,1)$ and

$$
\liminf _{n \rightarrow+\infty} F_{n}\left(u_{n}\right) \geq F(u)
$$

Proof. The key point of the proof is the construction of a suitable sequence of functions $v_{n}: \frac{1}{n} \mathbb{Z}^{2} \rightarrow\{0,1\}$ such that for any $\varrho>0$ and for a suitable choice of $\eta$ we have

$$
F_{n}\left(u_{n} ;(\varrho, 1-\varrho)\right) \geq E_{n}\left(v_{n} ; Q_{\varrho}^{\eta}\right)
$$

provided that $n>\frac{1}{\varrho}$, where $Q_{\varrho}^{\eta}=(\varrho, 1-\varrho) \times(-\eta, 1-\eta), E_{n}$ is the nearest-neighbour interaction energy defined in (19), and for $A \subset(0,1] F_{n}(\cdot ; A)$ is the localized version of $F_{n}$ defined by

$$
F_{n}(u ; A)=\frac{1}{n} \sum_{\{i, j\} \in \mathcal{E}_{n}(A)}\left(u_{i}-u_{j}\right)^{2}
$$

with

$$
\mathcal{E}_{n}(A)=\left\{\{i, j\}: i, j \in \mathbb{N} \cap n^{2} A \text { and }|i-j|=1 \text { or }|i-j|=n\right\} .
$$


Let $\left\{u_{n}\right\}$ be such that $u_{n} \in \mathcal{A}_{n}$ and $F_{n}\left(u_{n}\right) \leq c<+\infty$. Since $\left\{u_{n}\right\}$ is equibounded in $L^{\infty}(0,1)$ we can assume that (up to subsequences) $u_{n} \stackrel{*}{\rightarrow} u$ in $L^{\infty}(0,1)$. Denoting for all $j=1, \ldots, n$ by $\alpha_{n}^{j}$ the integral mean

$$
\alpha_{n}^{j}=n \int_{I_{n}^{j}} u_{n}(t) d t=\frac{1}{n} \sum_{k=1}^{n} u_{n}\left(\frac{j-1}{n}+\frac{k}{n^{2}}\right),
$$

where $I_{n}^{j}=\left(\frac{j-1}{n}, \frac{j}{n}\right]$, we define $\hat{u}_{n}$ in each $I_{n}^{j}$ by setting

$$
\hat{u}_{n}= \begin{cases}1 & \text { in }\left(\frac{j-1}{n}, \frac{j-1}{n}+\frac{\alpha_{n}^{j}}{n}\right] \\ 0 & \text { in }\left(\frac{j-1}{n}+\frac{\alpha_{n}^{j}}{n}, \frac{j}{n}\right]\end{cases}
$$

if $0<\alpha_{n}^{j}<1$, and $\hat{u}_{n}=u_{n}$ otherwise (see Fig. 3).
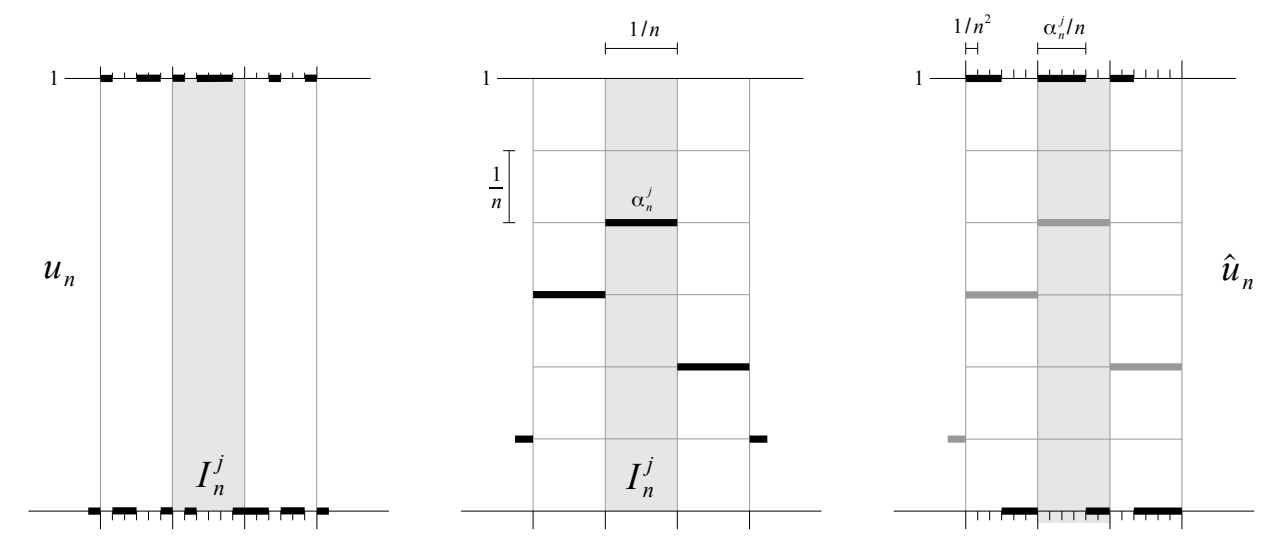

Figure 3: Construction of test functions for the lower bound

Note that in each $I_{n}^{j}$ this construction corresponds to setting $\hat{u}_{n}=1$ in the first $n \alpha_{n}^{j}$ points of $I_{n}^{j} \cap\left(\frac{1}{n^{2}} \mathbb{N}\right)$, and 0 in the remaining $n-n \alpha_{n}^{j}$. By construction, it follows that $\hat{u}_{n}$ weakly* converges in $L^{\infty}(0,1)$ to the same limit $u$, and

$$
F_{n}\left(u_{n}\right) \geq F_{n}\left(\hat{u}_{n}\right) .
$$

Indeed, with regard to the nearest-neighbour connections, since $\hat{u}_{n}$ has at most one jump in $\left(\frac{j-1}{n}, \frac{j}{n}\right)$, then, denoted by $S(v)$ the set of discontinuity points of a function $v$, $\# S\left(\hat{u}_{n}\right)=\min \left\{\# S\left(u_{n}\right), 2\right\}$ in each interval $I_{n}^{j}$ for $j<n$, and $\# S\left(\hat{u}_{n}\right)=\min \left\{\# S\left(u_{n}\right), 1\right\}$ in $I_{n}^{n}$. Concerning the long range interactions, for each pair of adjacent intervals $I_{n}^{j-1}$, $I_{n}^{j}$ the energy $F_{n}\left(u_{n}\right)$ counts the number of points in the symmetric difference $(n+$ $\left.K_{j-1}\left(u_{n}\right)\right) \triangle K_{j}\left(u_{n}\right)$, where $K_{j}(u)=\left\{i: u_{i}=1, \frac{i}{n^{2}} \in I_{n}^{j}\right\}$. The inequality (21) follows 
by noting that

$$
\begin{aligned}
\#\left(\left(n+K_{j-1}\left(u_{n}\right)\right) \triangle K_{j}\left(u_{n}\right)\right) & \geq\left|\#\left(n+K_{j-1}\left(u_{n}\right)\right)-\# K_{j}\left(u_{n}\right)\right| \\
& =\#\left(\left(n+K_{j-1}\left(\hat{u}_{n}\right)\right) \triangle K_{j}\left(\hat{u}_{n}\right)\right) .
\end{aligned}
$$

Now, we define the set $G_{n}=\bigcup_{j=1}^{n} R_{n}^{j}$ where $R_{n}^{j}=\left[\frac{j-1}{n}, \frac{j}{n}\right] \times\left[0, \alpha_{n}^{j}\right]$ for any $j=1, \ldots, n$. By construction, in each $Q_{n}(j, k)=\left(\frac{j-1}{n}, \frac{j}{n}\right] \times\left(\frac{k-1}{n}, \frac{k}{n}\right]$ we have $\chi_{G_{n}}=\hat{u}_{n}\left(\frac{j-1}{n}+\frac{k}{n^{2}}\right)$. Hence, with fixed $\varrho>0$, for all $n>\frac{1}{\varrho}$

$$
\begin{aligned}
F_{n}\left(\hat{u}_{n}\right) & \geq \frac{1}{n} \sum_{\left\{z, z^{\prime}\right\} \in \mathcal{N}(0, n]^{2}}\left(\left(\chi_{G_{n}}\right)_{z}-\left(\chi_{G_{n}}\right)_{z^{\prime}}\right)^{2} \\
& \geq \frac{1}{n} \sum_{\left\{z, z^{\prime}\right\} \in \mathcal{N}\left(n Q_{\varrho}^{0}\right)}\left(\left(\chi_{G_{n}}\right)_{z}-\left(\chi_{G_{n}}\right)_{z^{\prime}}\right)^{2}=E_{n}\left(\chi_{G_{n}} ; Q_{\varrho}^{0}\right)
\end{aligned}
$$

Then, recalling Proposition 2 , since $E_{n}\left(\chi_{G_{n}} ; Q_{\varrho}^{0}\right)=\mathcal{H}^{1}\left(\partial G_{n} \cap Q_{\varrho}^{0}\right)$, by compactness we deduce that (up to subsequences) $G_{n}$ converges in measure to a set of finite perimeter $G \subset Q_{\varrho}^{0}$.

In order to optimize the lower estimate, we also have to consider the part of the energy $F_{n}\left(\hat{u}_{n}\right)$ that comes from the interactions between $\frac{j}{n}$ and $\frac{j}{n}+\frac{1}{n^{2}}$ for $j=1, \ldots, n-$ 1 , corresponding to the length of the intersection of $\{y=0\}$ with the boundary of the periodic extension of $G_{n}$ to $(0,1)^{2}+(1 / n,-1)$ in $(0,1)^{2} \cup\left((0,1)^{2}+(1 / n,-1)\right.$. Setting $\tilde{G}_{n}=G_{n} \cup\left(G_{n}+(1 / n,-1)\right.$, for any $\eta \in(0,1)$ we have the estimate

$$
F_{n}\left(\hat{u}_{n}\right) \geq E_{n}\left(\chi_{\tilde{G}_{n}} ; Q_{\varrho}^{\eta}\right)
$$

Note that $\tilde{G}_{n}$ converges in measure to $\tilde{G}=G \cup(G+(0,-1))$ in $Q_{\varrho}^{0} \cup\left(Q_{\varrho}^{0}+(0,-1)\right)$. Since we can find $\eta \in(0,1)$ such that

$$
\mathcal{H}^{1}\left(\partial^{*} G \cap\{y=1-\eta\}\right)=0
$$

as $G$ is a set of finite perimeter, then

$$
\mathcal{H}^{1}\left(\partial^{*} \tilde{G} \cap Q_{\varrho}^{\eta}\right)=\mathcal{H}^{1}\left(\partial^{*} G \cap Q_{\varrho}^{0}\right)+\mathcal{H}^{1}\left(\partial^{*} G \cap\{y=0\} \cap Q_{\varrho}^{0}\right) .
$$

Hence, for such $\eta$, by applying the lower estimate for $E_{n}$ given by Proposition 2 we get

$$
\begin{aligned}
\liminf _{n \rightarrow+\infty} E_{n}\left(\chi_{\tilde{G}_{n}} ; Q_{\varrho}^{\eta}\right) & \geq \int_{\partial^{*} \tilde{G} \cap Q_{\varrho}^{\eta}}\left\|\nu_{\tilde{G}}\right\|_{1} d \mathcal{H}^{1} \\
& =\int_{\partial^{*} G \cap Q_{\varrho}^{0}}\left\|\nu_{G}\right\|_{1} d \mathcal{H}^{1}+\mathcal{H}^{1}\left(\partial^{*} G \cap\{y=0\} \cap Q_{\varrho}^{0}\right) .
\end{aligned}
$$


Now, we show that the weak* limit $u$ of $u_{n}$ belongs to $B V$, and $G$ is in fact its subgraph. We start by considering the piecewise constant function $\bar{u}_{n}$ defined by setting

$$
\bar{u}_{n}(x)=n \int_{I_{n}^{j}} \hat{u}_{n}(t) d t=n \int_{I_{n}^{j}} u_{n}(t) d t \quad \text { for } x \in I_{n}^{j}
$$

for each $j$. By construction, the set $G_{n}$ is the subgraph of $\bar{u}_{n}$; that is, $(x, y) \in G_{n}$ if and only if $0 \leq y \leq \bar{u}_{n}(x)$. Moreover, $\left\{u_{n}\right\}$ is a Cauchy sequence in $L^{1}(\varrho, 1-\varrho)$ since $G_{n}$ converges in measure. By construction, $\bar{u}_{n} \stackrel{*}{\rightarrow} u$ in $L^{\infty}(0,1)$, hence $\bar{u}_{n} \rightarrow u$ in $L^{1}(\varrho, 1-\varrho)$. Since $G_{n}$ is the subgraph of $\bar{u}_{n}$, the pointwise almost-everywhere convergence of $\bar{u}_{n}$ ensures that $(x, y) \in G$ if and only if $0 \leq y \leq \bar{u}(x)$ almost everywhere in $Q_{\varrho}^{0}$.

Since $G$ has finite perimeter, $u$ is a $B V$ function. Moreover, since

$$
\mathcal{H}^{1}\left(\partial^{*} G \cap\{y=0\} \cap Q_{\varrho}^{0}\right)=\mathcal{H}^{1}(\{x \in(\varrho, 1-\varrho): 0<u(x)<1\}),
$$

we have

$$
\begin{aligned}
\int_{\partial^{*} G \cap Q_{\varrho}^{0}} & \left\|\nu_{G}\right\|_{1} d \mathcal{H}^{1}+\mathcal{H}^{1}\left(\partial^{*} G \cap\{y=0\} \cap Q_{\varrho}^{0}\right) \\
& =\mathcal{H}^{1}(\{x \in(\varrho, 1-\varrho): 0<u(x)<1\})+|D u|(\varrho, 1-\varrho) \\
& =F(u ;(\varrho, 1-\varrho)) .
\end{aligned}
$$

Indeed, note that

$$
\nu_{G}(x, y)=\frac{\left(u^{\prime}, 1\right)}{\sqrt{1+\left|u^{\prime}\right|^{2}}} \quad x \text {-a.e. } \quad \text { and } \quad \nu_{G}(x, y)=(1,0) \frac{D_{s} u}{\left|D_{s} u\right|} \quad\left|D_{s} u\right| \text {-a.e. }
$$

so that

$$
\begin{aligned}
\int_{\partial^{*} G \cap Q_{\varrho}^{0}}\left\|\nu_{G}\right\|_{1} d \mathcal{H}^{1}+\mathcal{H}^{1}(\{x \in(\varrho, 1-\varrho): u(x) \in\{0,1\}\}) \\
=\int_{\varrho}^{1-\varrho} \frac{1+\left|u^{\prime}\right|}{\sqrt{1+\left|u^{\prime}\right|^{2}}} \sqrt{1+\left|u^{\prime}\right|^{2}} d x+\int_{(\varrho, 1-\varrho)}\left|D_{s} u\right| \\
=|D u|(\varrho, 1-\varrho)+\mathcal{H}^{1}(\varrho, 1-\varrho) .
\end{aligned}
$$

Hence, by estimates (21), (22), (24) and (25), we get the liminf inequality

$$
\liminf _{n \rightarrow+\infty} F_{n}\left(u_{n}\right) \geq F(u ;(\varrho, 1-\varrho))
$$

for any $\varrho>0$. 
Proposition 5 (Upper bound). Given $u \in B V(0,1)$ with values in $[0,1]$ there exists a recovery sequence $\left\{u_{n}\right\}$ such that $u_{n} \in \mathcal{A}_{n}, u_{n} \stackrel{*}{\rightarrow} u$ in $L^{\infty}(0,1)$ and

$$
\limsup _{n \rightarrow+\infty} F_{n}\left(u_{n}\right) \leq F(u)
$$

Proof. As a first step, we show that it is sufficient to prove the limsup inequality for $u$ piecewise constant. To this end, we first use a mollification argument. We choose $\eta>0$ such that both $\eta$ and $1-\eta$ are approximate continuity points for $u$, and we extend $u$ by reflection in $(-\eta, 0)$ and $(1,1+\eta)$; namely,

$$
u(x)=u(-x) \quad \text { if } x \in(-\eta, 0) \quad \text { and } \quad u(x)=u(2-x) \quad \text { if } x \in(1,1+\eta) .
$$

Denoting this extension by $u^{\eta}:(-\eta, 1+\eta) \rightarrow \mathbb{R}$ it follows that

$$
\left|D u^{\eta}\right|(-\eta, 1+\eta) \leq|D u|(0,1)+o(1)_{\eta \rightarrow 0} .
$$

Now, let $\left\{\varrho_{\varepsilon}\right\}$ be a sequence of smooth convolution kernels such that, for any $\varepsilon>0$, $\int_{\mathbb{R}} \varrho_{\varepsilon}(t) d t=1$ and $\operatorname{supp}\left(\varrho_{\varepsilon}\right)=[-\varepsilon, \varepsilon]$; setting $u_{\varepsilon}^{\eta}=u_{\eta} * \varrho_{\varepsilon}$, for $\varepsilon<\eta$ we get the estimate

$$
\left|D u_{\varepsilon}^{\eta}\right|(0,1) \leq\left|D u^{\eta}\right|(-\varepsilon, 1-\varepsilon) \leq\left|D u^{\eta}\right|(-\eta, 1-\eta) .
$$

Since we need to estimate the measure of the set where the values of each function belong to $(0,1)$, we set for any $\delta \in(0,1)$

$$
u_{\varepsilon}^{\eta, \delta}=\left(\left(\left(u_{\varepsilon}^{\eta}-\frac{1}{2}\right)(1+\delta)+\frac{1}{2}\right) \vee 0\right) \wedge 1
$$

with this definition, $0<u_{\varepsilon}^{\eta, \delta}<1$ implies $\frac{\delta}{4} \leq u_{\varepsilon}^{\eta} \leq 1-\frac{\delta}{4}$; then, the properties of the convolution ensure that

$$
\begin{aligned}
\left|\left\{x \in(0,1): 0<u_{\varepsilon}^{\eta, \delta}<1\right\}\right| & \leq\left|\left\{x \in(0,1): \frac{\delta}{4} \leq u_{\varepsilon}^{\eta} \leq 1-\frac{\delta}{4}\right\}\right| \\
& \leq\left|\left\{x \in(0,1): 0<u^{\eta}<1\right\}\right|+2 \varepsilon .
\end{aligned}
$$

Since

$$
\left|D u_{\varepsilon}^{\eta, \delta}\right|(0,1) \leq(1+\delta)\left|D u_{\varepsilon}^{\eta}\right|(0,1),
$$

recalling (28), (29) and (30) we get

$$
F\left(u_{\varepsilon}^{\eta, \delta}\right) \leq(1+\delta)\left(F(u)+o(1)_{\eta \rightarrow 0}\right)+2 \varepsilon .
$$

Hence for any $\sigma>0$ we can find $\eta, \varepsilon$ and $\delta$ small enough to have $\left\|u-u_{\varepsilon}^{\eta, \delta}\right\|_{L^{1}}<\sigma$ and

$$
F\left(u_{\varepsilon}^{\eta, \delta}\right) \leq F(u)+\sigma .
$$


Now we construct a sequence $\left\{u_{k}\right\}$ of piecewise-constant functions (where we omit the dependence on $\eta, \varepsilon$ and $\delta$ ) such that $u_{k} \rightarrow u_{\varepsilon}^{\eta, \delta}$ in $L^{1}(0,1)$ as $k \rightarrow+\infty$ and

$$
F\left(u_{k}\right) \leq F\left(u_{\varepsilon}^{\eta, \delta}\right) .
$$

For any fixed integer $k \geq 1$ we consider the intervals $J_{k}^{i}=\left(\frac{i-1}{k}, \frac{i}{k}\right]$; since $u_{\varepsilon}^{\eta, \delta} \in C^{0}[0,1]$, for $k$ large enough either $J_{k}^{i} \cap\left\{u_{\varepsilon}^{\eta, \delta}=0\right\}=\emptyset$ or $J_{k}^{i} \cap\left\{u_{\varepsilon}^{\eta, \delta}=1\right\}=\emptyset$ for any $i=1, \ldots, k$. Hence, for such $k$ we can define $u_{k}$ by setting in each $J_{k}^{i}$

$$
u_{k}= \begin{cases}0 & \text { if } J_{k}^{i} \cap\left\{u_{\varepsilon}^{\eta, \delta}=0\right\} \neq \emptyset \\ 1 & \text { if } J_{k}^{i} \cap\left\{u_{\varepsilon}^{\eta, \delta}=1\right\} \neq \emptyset \\ u_{\varepsilon}^{\eta, \delta}\left(\frac{i}{k}\right) & \text { if } J_{k}^{i} \subset\left\{0<u_{\varepsilon}^{\eta, \delta}<1\right\} .\end{cases}
$$

Note that the uniform continuity of $u_{\varepsilon}^{\eta, \delta}$ ensures the uniform convergence of $u_{k} \rightarrow u_{\varepsilon}^{\eta, \delta}$, hence $u_{k} \rightarrow u_{\varepsilon}^{\eta, \delta}$ in $L^{1}(0,1)$. By construction we have that for $k$ large enough

$$
\mathcal{H}^{1}\left(\left\{0<u_{k}<1\right\}\right) \leq \mathcal{H}^{1}\left(\left\{0<u_{\varepsilon}^{\eta, \delta}<1\right\}\right), \quad\left|D u_{k}\right|(0,1) \leq\left|D u_{\varepsilon}^{\eta, \delta}\right|(0,1),
$$

hence (32) holds and it is sufficient to prove the limsup-inequality for $u$ piecewise constant (see [5] Remark 1.29).
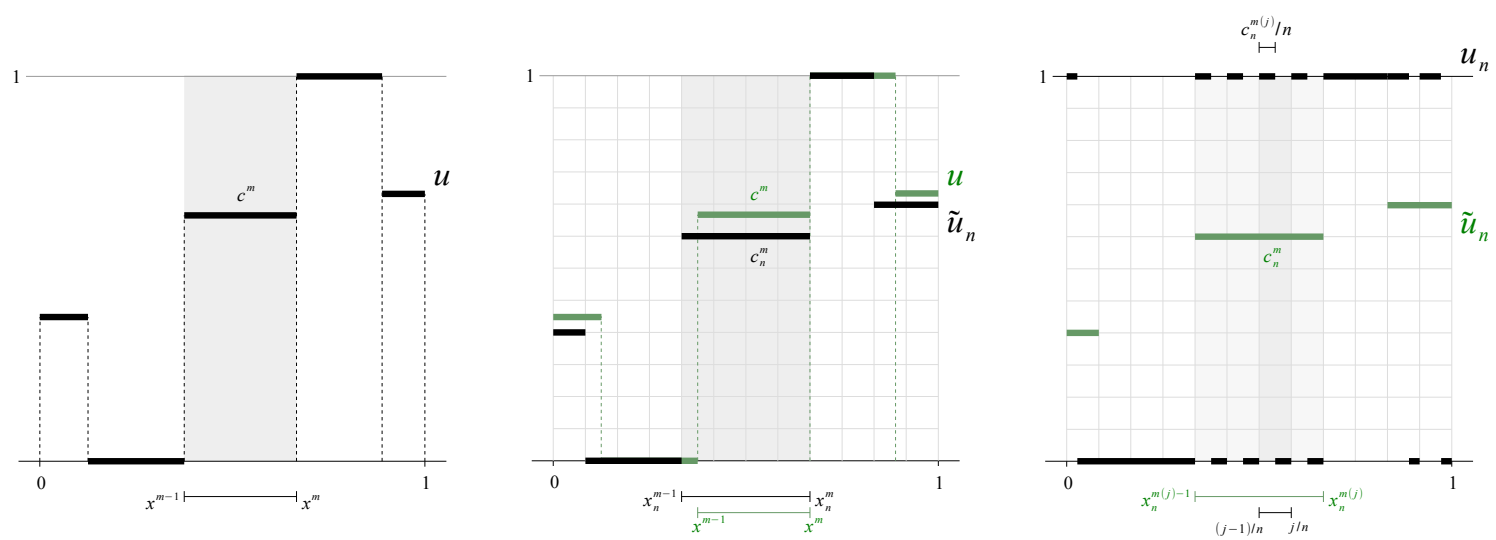

Figure 4: Construction of a recovery sequence

Let $u$ be a piecewise-constant function given by the partition $\left\{x^{m}\right\}_{m=0}^{k}$ and values $c^{m} \in[0,1]$ such that $u=c^{m}$ in $\left(x^{m-1}, x^{m}\right)$ for $m=1, \ldots, k$ (see the first picture in Fig. 4). For each $m=0, \ldots k$ we set $x_{n}^{m}=\frac{\left\lfloor n x^{m}\right\rfloor}{n}$; we define $\tilde{u}_{n}$ by setting $\tilde{u}_{n}=c_{n}^{m}=$ $\frac{\left\lfloor n c^{m}\right\rfloor}{n}$ in each interval $\left(x_{n}^{m-1}, x_{n}^{m}\right)$ (see the second picture in Fig. 4). In this way we get the inequality

$$
F\left(\tilde{u}_{n}\right) \leq F(u)+\frac{k}{n}
$$


Now, we can construct the recovery sequence $u_{n}$ similarly as we did in (20) in the proof of the liminf inequality. For each $j$ let $m(j) \in\{1, \ldots, k\}$ be such that $\left(\frac{j-1}{n}, \frac{j}{n}\right) \subset$ $\left(x_{n}^{m(j)-1}, x_{n}^{m(j)}\right)$; we define $u_{n}$ in $\left(\frac{j-1}{n}, \frac{j}{n}\right]$ by setting

$$
u_{n}= \begin{cases}1 & \text { in }\left(\frac{j-1}{n}, \frac{j-1}{n}+\frac{c_{n}^{m(j)}}{n}\right] \\ 0 & \text { in }\left(\frac{j-1}{n}+\frac{c_{n}^{m(j)}}{n}, \frac{j}{n}\right]\end{cases}
$$

if $c_{n}^{m(j)} \neq 0,1, u_{n}=0$ if $c_{n}^{m(j)}=0$ and $u_{n}=1$ if $c_{n}^{m(j)}=1$ (see the third picture in Fig. 4).

Note that $u_{n} \stackrel{*}{\rightarrow} u$ in $L^{\infty}(0,1)$. By construction $F_{n}\left(u_{n}\right)=F\left(\tilde{u}_{n}\right)$, hence

$$
\limsup _{n \rightarrow+\infty} F_{n}\left(u_{n}\right) \leq F(u)
$$

after recalling (33).

\section{Volume-constrained minimization problems}

In this section we examine the behaviour of functionals $F_{n}$ subjected to the constraint of fixing the number of $i$ such that $u_{i}=1$. Since the form of the minimizers of such a constraint depends on the size of the domain, we extend the previous result to functions defined on $[0, L]$ for any $L>0$.

\subsection{Compatibility of the volume constraint}

Let $k_{n}$ be a sequence of integers such that $0 \leq k_{n} \leq L n^{2}$ such that

$$
\lim _{n \rightarrow+\infty} \frac{k_{n}}{L n^{2}}=\sigma \in(0,1)
$$

Let $L_{n}=\frac{\left\lfloor L n^{2}\right\rfloor}{n^{2}}$ and $\mathcal{A}_{n}\left(L, k_{n}\right)$ be the set of the functions $u:\left(0, L_{n}\right] \rightarrow\{0,1\}$ with $u$ constant on each interval $\left(\frac{i-1}{n^{2}}, \frac{i}{n^{2}}\right], i=1, \ldots,\left\lfloor L n^{2}\right\rfloor$ and such that

$$
\#\left\{i \in\left\{1, \ldots,\left\lfloor L n^{2}\right\rfloor\right\}: u_{i}=1\right\}=k_{n},
$$

where $u_{i}$ stands for $u\left(\frac{i}{n^{2}}\right)$ as previously. Such a $u$ corresponds to a discrete function, which we still denote by $u$, defined as its restriction to $\left(\frac{1}{n^{2}} \mathbb{N}\right) \cap(0, L]$. If necessary we extend each such function to 0 outside $\left(0, L_{n}\right]$. Such an extension does not modify the energies we are going to consider and makes convergence statements easier.

We set

$$
\mathcal{E}_{n}(L)=\left\{\{i, j\}: i, j \in \mathbb{N} \cap\left(0, L n^{2}\right] \text { and }|i-j|=1 \text { or }|i-j|=n\right\} .
$$


For $u \in \mathcal{A}_{n}\left(L, k_{n}\right)$ we define the functional

$$
F_{n}^{L, k_{n}}(u)=\frac{1}{n} \sum_{\{i, j\} \in \mathcal{E}_{n}(L)}\left(u_{i}-u_{j}\right)^{2} .
$$

Theorem 6 (compatibility of volume constraints). The sequence of functionals $\left\{F_{n}^{L, k_{n}}\right\}$ $\Gamma$-converges with respect to the weak-* convergence in $L^{\infty}(0, L)$ to the functional $F^{L, \sigma}$ : $L^{\infty}(0, L) \rightarrow[0,+\infty]$ given by

$$
F^{L, \sigma}(u)=\left\{\begin{array}{l}
2 \mathcal{H}^{1}(\{x \in(0, L): 0<u(x)<1\})+|D u|(0, L) \\
+\infty \quad \begin{array}{l}
\text { if } u \in B V(0, L), 0 \leq u \leq 1, \int_{0}^{L} u d x=L \sigma \\
\text { otherwise. }
\end{array}
\end{array}\right.
$$

Proof. Since functions satisfying the integral constraint (35) converge to functions satisfying $\int_{0}^{L} u d x=L \sigma$, the liminf inequality is immediately satisfied. It remains to prove the existence of a recovery sequence for $u$ in the domain of $F^{L, \sigma}$. By a density argument, it suffices to treat the case of $u$ piecewise constant since the construction in the proof of Proposition 5 is compatible with the integral constraint. Moreover, we may reduce to treat the case of $u$ constant, since the construction below is immediately extended to a piecewise-constant $u$.

We now exhibit a recovery sequence for the constant target function $u=\sigma$ in $(0, L)$.

Writing $k_{n}=(\lfloor L n\rfloor+1) a_{n}+b_{n}$ with $a_{n} \in\{0, \ldots, n\}$ and $b_{n} \in\{0, \ldots,\lfloor L n\rfloor\}$, we construct a function $v_{n}$ defined in $\left(0, \frac{\lfloor L n\rfloor+1}{n}\right]$ as follows. We denote by $\lambda_{n}$ the number $\left\lfloor L n^{2}\right\rfloor-n\lfloor L n\rfloor$ (measuring the 'defect of periodicity' of the interval $[0, L]$ ) and define $v_{n}$ as follows.

- If $a_{n} \leq \lambda_{n}$ then we have two constructions, according to $b_{n}$ : in each $\left(\frac{j-1}{n}, \frac{j}{n}\right]$ with $b_{n} \geq j$ we set

$$
v_{n}= \begin{cases}1 & \text { in }\left(\frac{j-1}{n}, \frac{j-1}{n}+\frac{a_{n}+1}{n^{2}}\right] \\ 0 & \text { in }\left(\frac{j-1}{n}+\frac{a_{n}+1}{n^{2}}, \frac{j}{n}\right] ;\end{cases}
$$

in each $\left(\frac{j-1}{n}, \frac{j}{n}\right]$ with $b_{n}<j$ we set

$$
v_{n}= \begin{cases}1 & \text { in }\left(\frac{j-1}{n}, \frac{j-1}{n}+\frac{a_{n}}{n^{2}}\right] \\ 0 & \text { in }\left(\frac{j-1}{n}+\frac{a_{n}}{n^{2}}, \frac{j}{n}\right] ;\end{cases}
$$

- If $\lambda_{n}<a_{n}<n$, then we write $a_{n}-\lambda_{n}+b_{n}=\gamma_{n}\lfloor L n\rfloor+\delta_{n}$, with $\delta_{n}<\lfloor L n\rfloor$, and the constructions are as follows: if $\delta_{n} \geq j$ we set

$$
v_{n}= \begin{cases}1 & \text { in }\left(\frac{j-1}{n}, \frac{j-1}{n}+\frac{a_{n}+\gamma_{n}+1}{n}\right] \\ 0 & \text { in }\left(\frac{j-1}{n}+\frac{a_{n}+\gamma_{n}+1}{n^{2}}, \frac{j}{n}\right]\end{cases}
$$


and if $\delta_{n}<j$

$$
v_{n}= \begin{cases}1 & \text { in }\left(\frac{j-1}{n}, \frac{j-1}{n}+\frac{a_{n}+\gamma_{n}}{n^{2}}\right] \\ 0 & \text { in }\left(\frac{j-1}{n}+\frac{a_{n}+\gamma_{n}}{n^{2}}, \frac{j}{n}\right]\end{cases}
$$

Now, we define $u_{n}$ as the restriction of $v_{n}$ to $\left(0, L_{n}\right]$. Note that $\#\left\{i \in\left\{1, \ldots,\left\lfloor L n^{2}\right\rfloor\right\}\right.$ : $\left.\left(u_{n}\right)_{i}=1\right\}=k_{n}$, that is $u_{n} \in \mathcal{A}\left(L, k_{n}\right)$.

Since $a_{n} \neq 0$ for $n$ large enough, the number of $\{i, j\}$ such that $|i-j|=1$ and $\left(u_{n}\right)_{i} \neq\left(u_{n}\right)_{j}$ is bounded by $2(\lfloor L n\rfloor+1)$, and the number of jumps between points at distance $n$ is at most 1 . Hence

$$
F_{n}^{L, k_{n}}\left(u_{n}\right) \leq \frac{1}{n}(2\lfloor L n\rfloor+3)=2 L+o(1)_{n \rightarrow+\infty}=F^{L, \sigma}(u)+o(1)_{n \rightarrow+\infty} .
$$

\subsection{A size effect}

As a consequence of Theorem 6, minimum problems for $F_{n}^{L, k_{n}}$ (i.e., volume-constrained minimum problems for $F_{n}$ on $\left.[0, L]\right)$ converge to the minimum problem

$$
\begin{aligned}
\min \left\{2 \mathcal{H}^{1}(\{x \in(0, L): 0<u(x)<1\})+|D u|(0, L):\right. & \\
& \left.u \in B V((0, L) ;[0,1]), \int_{0}^{L} u d x=\sigma L\right\} .
\end{aligned}
$$

This problem can be simplified by remarking that

- if $u$ takes the values 1 and 0 on a set of non-zero measure then $\mathcal{H}^{1}(\{x \in(0, L)$ : $0<u(x)<1\})=0$. Indeed if $u$ takes the values 1 and 0 then $|D u| \geq 1$, and the function $v(x)=\chi_{[0, \sigma L]}$ has a lower energy than $u$;

- we may assume that $\{x \in(0, L): 0<u(x)<1\}$ is an interval and $u$ is constant on $\{x \in(0, L): 0<u(x)<1\}$.

We end up with four cases:

A) the minimum is $u_{\min }=\sigma$. In this case, $F^{L, \sigma}\left(u_{\min }\right)=2 L$;

B) the minimum is $u_{\min }=\chi_{[0, \sigma L]}$. In this case, $F^{L, \sigma}\left(u_{\min }\right)=1$;

C) the minimum is obtained by minimizing on functions of the form $u=\frac{\sigma L}{y} \chi_{[0, y]}$. In this case, $F^{L, \sigma}\left(u_{\min }\right)=2 \sqrt{2 \sigma L}$;

D) the minimum is obtained by minimizing on functions of the form $u=1-$ $\frac{(1-\sigma) L}{y} \chi_{[0, \sigma y]}$. In this case, $F^{L, \sigma}\left(u_{\min }\right)=2 \sqrt{2(1-\sigma) L}$. 


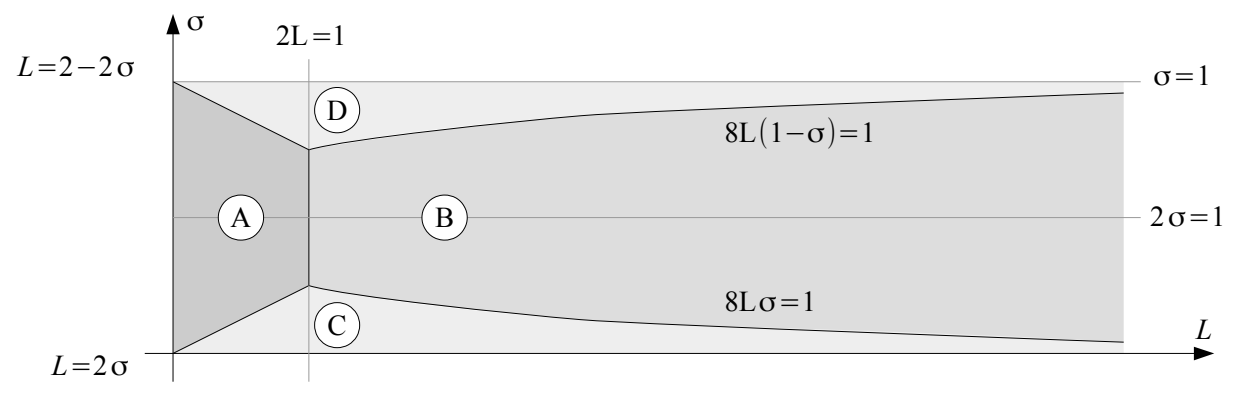

Figure 5: Description of minimizers

In addition, note that the behaviour of the minimum problems is the same for $\sigma$ and $1-\sigma$, so that it is sufficient to examine the case $\sigma \leq 1 / 2$. An explicit calculation yields the analysis highlighted in Fig. 5.

Remark 7 (size effect for volume-constrained minimization). As remarked above, it is not restrictive to limit our description to $\sigma \leq 1 / 2$. We have two different behaviours depending on $L$.

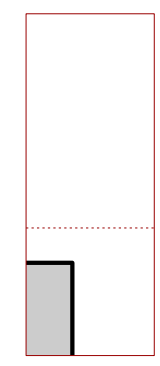

$\sigma<\frac{L}{2}$

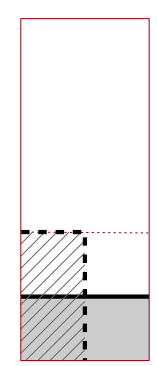

$\sigma=\frac{L}{2}$

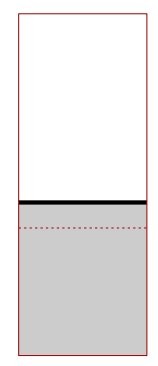

$\frac{L}{2}<\sigma<1-\frac{L}{2}$

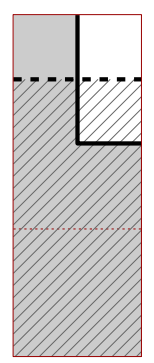

$\sigma=1-\frac{L}{2}$

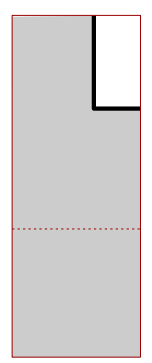

$\sigma>1-\frac{L}{2}$

Figure 6: Evolution of the form of the minimizers in small domains

Small-size domain: if $L<\frac{1}{2}$ then we have

i) for $\sigma<\frac{L}{2}$ the minimizers are

$$
u_{1}=\sqrt{2 \sigma L} \chi_{\left[0, \frac{1}{2} \sqrt{2 \sigma L}\right]}, \quad u_{2}=\sqrt{2 \sigma L} \chi_{\left[L-\frac{1}{2} \sqrt{2 \sigma L}, L\right]}
$$

ii) for $\sigma=\frac{L}{2}$ the minimizers are the constant $u=\sigma$ and the two functions

$$
u_{1}=L \chi_{[0, L / 2]}, \quad u_{2}=L \chi_{[L / 2, L]}
$$


iii) for $\frac{L}{2}<\sigma$ the minimizer is the constant $u=\sigma$.

In Fig. 6 we picture two-dimensional sets corresponding to minimizers of the energy at varying $\sigma$. The length of the part of the sets highlighted in the figure gives the corresponding value of the energy. Note that at $\sigma=\frac{L}{2}$ (and symmetrically at $\sigma=1-\frac{L}{2}$, we have a discontinuity in the form of minimizers.

Large-size domain: if $L>\frac{1}{2}$ then we have

i) for $\sigma<\frac{1}{8 L}$ the minimizers are as in case (C) above

$$
u_{1}=\sqrt{2 \sigma L} \chi_{\left[0, \frac{1}{2} \sqrt{2 \sigma L}\right]}, \quad u_{2}=\sqrt{2 \sigma L} \chi_{\left[L-\frac{1}{2} \sqrt{2 \sigma L}, L\right]}
$$

ii) for $\sigma=\frac{1}{8 L}$ the four minimizers are the functions

$$
u_{1}=\frac{1}{2} \chi_{\left[0, \frac{1}{4}\right]}, \quad u_{2}=\frac{1}{2} \chi_{\left[L-\frac{1}{4}, L\right]}, \quad u_{3}=\chi_{\left[0, \frac{1}{8}\right]}, \quad u_{4}=\chi_{\left[L-\frac{1}{8}, L\right]} ;
$$

iii) for $\sigma>\frac{1}{8 L}$ the minimizers are

$$
u_{1}=\chi_{[0, \sigma L]}, \quad u_{2}=\chi_{[(1-\sigma) L, L]} .
$$

In Fig. 7 we again picture minimizers at varying $\sigma$. Again, note the discontinuity in the form of the minimizers at $\sigma=\frac{1}{8 L}$ (and symmetrically at $\sigma=1-\frac{1}{8 L}$ ).
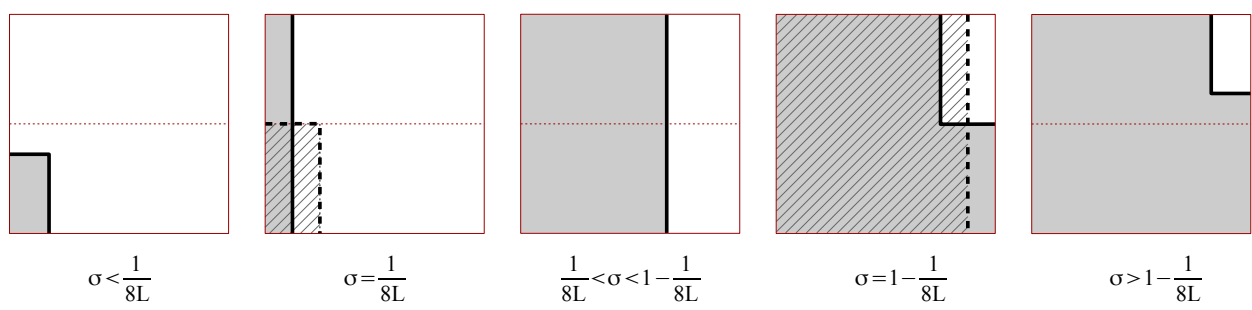

Figure 7: Evolution of the form of the minimizers in large domains

Remark 8. From the description of minimizers in the previous remark we easily derive the shape of minimizers for the discrete problems, given by the corresponding recovery sequences. A pictorial description for small-size domains is given in Fig. 1.

\section{Boundary effects in periodic minimization}

In this section we analyze the effect of periodic boundary conditions on the volumeconstrained minimization. The overall behaviour in dependence of $L$ and $\sigma$ will be 
described by introducing an additional parameter $\tau$, which quantifies the 'defect of $\frac{1}{n}$ periodicity' of the underlying domain $[0, L]$, and computing a $\tau$-depending $\Gamma$-limit. The existence of the $\Gamma$-limit only up to subsequences depending on $\tau$ is not an uncommon feature when studying fine effects of homogenization depending on boundary effects (see e.g. [13] Section 1.3).

We define the set of the periodic interactions as

$$
\mathcal{E}_{n}^{\#}(L)=\left\{\{i, j\}: i, j \in \mathbb{N} \cap\left(0,\left\lfloor L n^{2}\right\rfloor\right]:|i-j| \in\left\{1,\left\lfloor L n^{2}\right\rfloor-1, n,\left\lfloor L n^{2}\right\rfloor-n\right\}\right\} .
$$

For $u \in \mathcal{A}_{n}\left(L, k_{n}\right)$ (as previously defined) we set

$$
F_{n}^{\#}(u)=\frac{1}{n} \sum_{\{i, j\} \in \mathcal{E}_{n}^{\#}(L)}\left(u_{i}-u_{j}\right)^{2}
$$

(the dependences on $L$ and $k_{n}$ are omitted).

We consider the sequence $\lambda_{n}=\left\lfloor L n^{2}\right\rfloor-n\lfloor L n\rfloor \in[0, n)$. Let $\tau_{n_{m}}$ be a subsequence of $\tau_{n}=\frac{\lambda_{n}}{n}$ converging to $\tau \in[0,1]$; we compute the $\Gamma$-limit for the corresponding sequence $\left\{F_{n_{m}}^{\#}\right\}$, again denoted by $\left\{F_{n}^{\#}\right\}$.

Remark 9. We denote by $\tau(L)$ the set of the limits of converging subsequences of $\left\{\tau_{n}\right\}$. If $L=\frac{p}{q}, p, q \in \mathbb{N}$ co-primes, then $\tau(L)=\left\{\frac{k}{q}: k \in \mathbb{N}, k \leq q\right\}$. Otherwise, if $L \notin \mathbb{Q}$ then $\tau(L)=[0,1]$. Indeed, the difference $\tau_{n}-(L n-\lfloor L n\rfloor)$ goes to 0 as $n \rightarrow+\infty$, and a theorem of Kronecker ensures that the sequence of the fractional parts of $L n$ is dense in $[0,1]$ if $L$ is not rational. Note that this implies that for any $\tau \in[0,1]$ the set of the values $L>0$ such that $\tau \in \tau(L)$ is dense in $(0,+\infty)$.

For $x, y \in[0,1]$ we define

$$
\phi_{\tau}(x, y)=\mathcal{H}^{1}\left([0,1] \cap\left(U_{x}^{\tau} \triangle U_{y}\right)\right)
$$

with $U_{y}=[0, y]$ and $U_{x}^{\tau}=[-\tau, x-\tau] \cup[1-\tau, x+1-\tau]$ (see Fig. 8).

Theorem 10. Let $\tau$ be defined as above, and $\phi_{\tau}$ the corresponding function in (41). The sequence of functionals $\left\{F_{n}^{\#}\right\} \Gamma$-converges with respect to the weak-* convergence in $L^{\infty}(0, L)$ to the functional $F_{\tau}^{\#}: L^{\infty}(0, L) \rightarrow[0,+\infty]$ given by

$$
F_{\tau}^{\#}(u)=\left\{\begin{array}{cc}
2 \mathcal{H}^{1}(\{x \in(0, L): 0<u<1\})+|D u|(0, L)+\phi_{\tau}\left(u\left(0^{+}\right), u\left(L^{-}\right)\right) \\
+\infty \quad \text { if } u \in B V(0, L), 0 \leq u \leq 1, \int_{0}^{L} u d x=L \sigma \\
\text { otherwise }
\end{array}\right.
$$




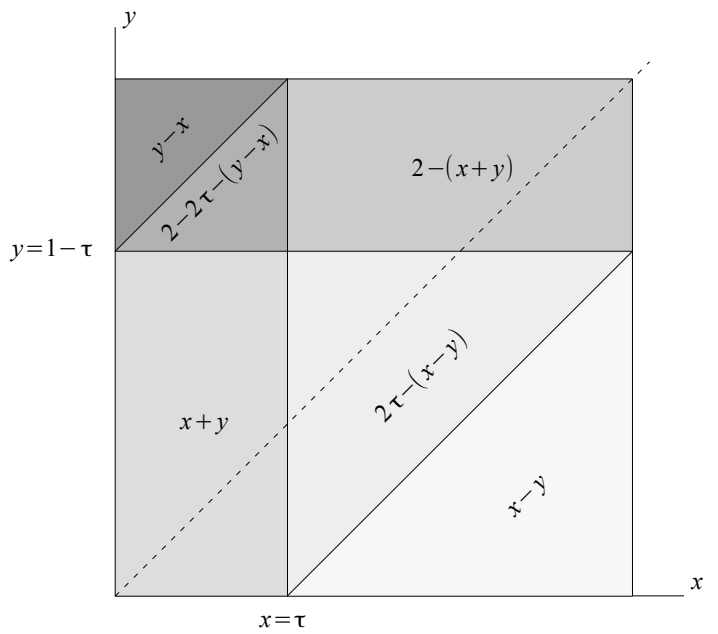

Figure 8: Pictorial description and values of $\phi_{\tau}$
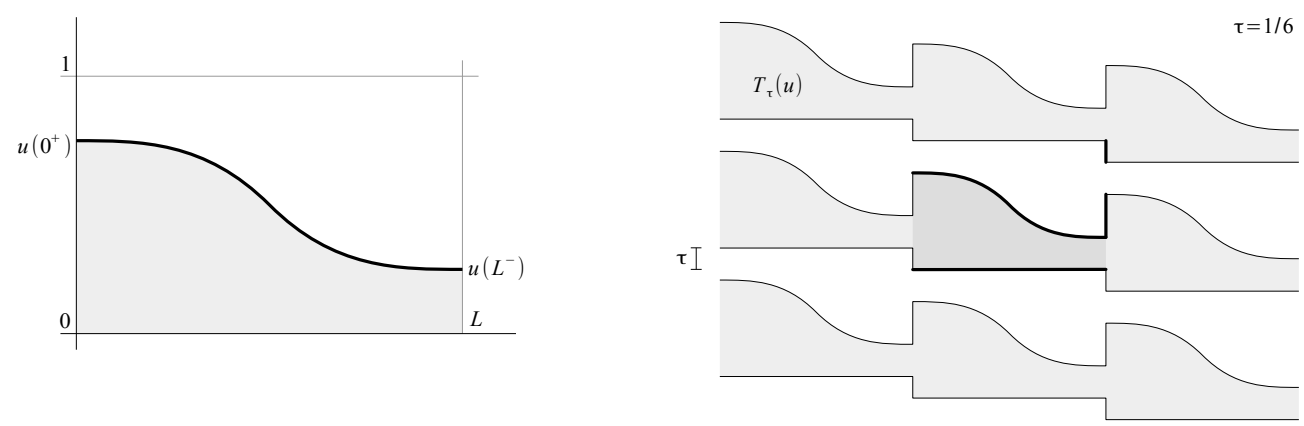

Figure 9: Description of $T_{\tau}(u)$

Remark 11 (Interpretation in terms of perimeters). Given $u \in B V((0, L) ;[0,1])$ and $\tau \in[0,1]$, we define the set $T_{\tau}(u) \subset \mathbb{R}^{2}$ as

$$
T_{\tau}(u)=\bigcup_{n_{1}, n_{2} \in \mathbb{Z}}\left(V(u)+\left(n_{1} L, n_{1}(1-\tau)+n_{2}\right)\right),
$$

where $V(u)=\{(x, y): 0 \leq x \leq L, 0 \leq y \leq u(x)\}$ is the subgraph of $u$ (see Fig. 9). Note that the value of the 1-perimeter of $T_{\tau}(u)$ in a periodicity cell equals $F_{\tau}^{\#}(u)$; i.e.,

$$
\begin{aligned}
\operatorname{Per}_{1}\left(T_{\tau}(u) ;(0, L] \times(0,1]\right)=2 \mathcal{H}^{1}( & \{x \in(0, L): 0<u<1\}) \\
& +|D u|(0, L)+\phi_{\tau}\left(u\left(0^{+}\right), u\left(L^{-}\right)\right) .
\end{aligned}
$$

The value of $\phi_{\tau}\left(u\left(0^{+}\right), u\left(L^{-}\right)\right)$is the measure of the part of the graph on the boundary of the periodicity cell highlighted in Fig. 9. 
Remark 12 (Properties of $\phi_{\tau}$ ). If $\tau=0$, then $\phi_{0}(x, y)=|x-y|$. Since $\phi_{\tau}(x, y)=$ $\phi_{1-\tau}(y, x)$, we can reduce to consider the case $\tau \leq \frac{1}{2}$. The following monotonicity property of $\phi_{\tau}$ will be useful in the computation of the minima of the functional $F^{\#}$ :

$$
\phi_{\tau}(x, y) \leq \phi_{\tau}(y, x) \quad \text { if } \quad x \geq y \quad \text { for } \quad \tau \leq \frac{1}{2} .
$$

Moreover, note that

$$
\phi_{\tau}(s, s)= \begin{cases}2 s & \text { if } s \leq \min \{\tau, 1-\tau\} \\ 2 \min \{\tau, 1-\tau\} & \text { if } \min \{\tau, 1-\tau\} \leq s \leq \max \{\tau, 1-\tau\} \\ 2(1-s) & \text { if } \max \{\tau, 1-\tau\} \leq s\end{cases}
$$

hence for the constant function $u=\sigma$ we get $F_{\tau}^{\#}(u)=2 \min \{\tau, 1-\tau, \sigma, 1-\sigma\}$.

Proof of the lower bound. Let $\left\{u_{n}\right\}$ be such that $u_{n} \in \mathcal{A}_{n}\left(L, k_{n}\right)$ and $F_{n}^{\#}\left(u_{n}\right) \leq c<$ $+\infty$. Since $\left\{u_{n}\right\}$ is equibounded in $L^{\infty}(0, L)$ we can assume that (up to subsequences) $u_{n} \stackrel{*}{\rightarrow} u$ in $L^{\infty}(0, L)$. Following the proof of Proposition 4 , we denote by $\alpha_{n}^{j}$ the integral mean of $u_{n}$ in $I_{n}^{j}$, where $I_{n}^{j}=\left(\frac{j-1}{n}, \frac{j}{n}\right\rfloor$ for $j<\lfloor L n\rfloor$ and $I_{n}^{\lfloor L n\rfloor}=\left(\frac{\lfloor L n\rfloor}{n}, L_{n}\right]$. Firstly, we define $\breve{u}_{n}$ in each $I_{n}^{j}$ with $j>1$ by setting

$$
\check{u}_{n}= \begin{cases}1 & \text { in }\left(\frac{j-1}{n}, \frac{j-1}{n}+\frac{\alpha_{n}^{j}}{n}\right] \\ 0 & \text { in }\left(\frac{j-1}{n}+\frac{\alpha_{n}^{j}}{n}, \frac{j}{n}\right]\end{cases}
$$

if $0<\alpha_{n}^{j}<1$, and $\check{u}_{n}=u_{n}$ otherwise. Note that this part of the construction the same as the one in definition (20). Now, we define $\check{u}_{n}$ in $I_{n}^{1}$ in order to minimize the $n$-range jumps in this interval. To this end, we have to set, whenever possible, $\left(\check{u}_{n}\right)_{i}=1$ if $i$ belongs to the set $\mathcal{I}^{1}$ of the indices such that $\left(\check{u}_{n}\right)_{i+n}=\left(\check{u}_{n}\right)_{i+\left\lfloor L n^{2}\right\rfloor-n}=1$ and $\left(\check{u}_{n}\right)_{i}=0$ if $i$ belongs to the set $\mathcal{I}^{0}$ of the indices such that $\left(\check{u}_{n}\right)_{i+n}=\left(\check{u}_{n}\right)_{i+\left\lfloor L n^{2}\right\rfloor-n}=0$. Note that, by construction, $\mathcal{I}^{1}$ has one of the three following forms: $\mathbb{Z} \cap\left(0, i^{\prime}\right]$ with $i^{\prime} \leq n \tau_{n}$, $\mathbb{Z} \cap\left(n \tau_{n}, i^{\prime \prime}\right]$ with $n \tau_{n}<i^{\prime \prime} \leq n$ or $\mathbb{Z} \cap\left(\left(0, i^{\prime}\right] \cup\left(n \tau_{n}, i^{\prime \prime}\right]\right)$. Similarly, the set $\mathcal{I}^{0}$ can be written as the union of at most two "intervals" $\mathbb{Z} \cap\left(j^{\prime}, n \tau_{n}\right]$ and $\mathbb{Z} \cap\left(j^{\prime \prime}, n\right]$. We define $\check{u}_{n}$ in $I_{n}^{1}$ by considering three cases. If $n \alpha_{n}^{1} \leq \# \mathcal{I}^{1}$, we set $\left(\check{u}_{n}\right)_{i}=1$ for the first $n \alpha_{n}^{1}$ indices $i$ in $\mathcal{I}^{1}$, and 0 otherwise. If $\# \mathcal{I}^{1}<n \alpha_{n}^{1} \leq n-\# \mathcal{I}^{0}$, then we define $\left(\check{u}_{n}\right)_{i}=1$ for any $i \in \mathcal{I}^{1}$ and for the first $n \alpha_{n}^{1}-\# \mathcal{I}^{1}$ points in the complementary set of $\mathcal{I}^{1} \cup \mathcal{I}^{0}$, and 0 otherwise. Finally, if $n-\# \mathcal{I}^{0}<n \alpha_{n}^{1}$, we define $\left(\check{u}_{n}\right)_{i}=1$ for any $i$ in the complementary set of $\mathcal{I}^{0}$ and in the first $n \alpha_{n}^{1}-\left(n-\# \mathcal{I}^{0}\right)$ points of $\mathcal{I}^{0}$, and 0 otherwise. The function $\check{u}_{n}$ belongs to $\mathcal{A}_{n}\left(L, k_{n}\right)$, and, following the idea of the proof of the estimate (21), from the construction of $\check{u}_{n}$ we deduce that

$$
F_{n}^{\#}\left(u_{n}\right) \geq F_{n}^{\#}\left(\check{u}_{n}\right)-\frac{c}{n}
$$


where $c$ is independent on $n$.

As before, we now construct a set $\tilde{G}_{n} \subset \mathbb{R}^{2}$ such that for $\eta>0$ we have

$$
F_{n}^{\#}\left(\check{u}_{n}\right) \geq E_{n}\left(\tilde{G}_{n} ; Q^{\eta}\right)
$$

where $E_{n}$ is the functional defined in $(19)$ and $Q^{\eta}=(\eta, L+\eta) \times(\eta, 1+\eta)$. Denoting by $\check{u}_{n}^{\#}$ the periodic extension to $\mathbb{R}$ of $\check{u}_{n}$, we set

$$
\chi_{G_{n}}=\check{u}_{n}^{\#}\left(\frac{j-1}{n}+\frac{k}{n^{2}}\right) \quad \text { in } \quad Q_{n}^{k, j}=\left(\frac{j-1}{n}, \frac{j}{n}\right] \times\left(\frac{k-1}{n}, \frac{k}{n}\right]
$$

with $j=1, \ldots,\lfloor L n\rfloor$ and $k=1, \ldots, n$. We set

$$
\tilde{G}_{n}=\bigcup_{n_{1}, n_{2} \in \mathbb{Z}}\left(G_{n}+\left(n_{1} \frac{\lfloor L n\rfloor}{n}, n_{1}\left(1-\tau_{n}\right)+n_{2}\right)\right) .
$$

The same argument as in the proof of Proposition 4 ensures that $G_{n}$ converges in measure to a set $G$ with finite perimeter in $Q^{0}$, which is in fact the subgraph $V(u)$ of the weak ${ }^{*}$-limit $u$ of $u_{n}$. Note that $u$ turns out to be $B V(0, L)$ with values in $[0,1]$, and $\int_{0}^{L} u d x=\sigma L$. Let $\eta \in(0,1)$ be such that

$$
\mathcal{H}^{1}\left(\partial^{*} T_{\tau}(u) \cap \partial Q^{\eta}\right)=0,
$$

where $T_{\tau}$ is defined in (43); hence, by Proposition 2

$$
\begin{aligned}
\liminf _{n \rightarrow+\infty} F_{n}^{\#}\left(u_{n}\right) & \geq \liminf _{n \rightarrow+\infty} F_{n}^{\#}\left(\check{u}_{n}\right) \geq \liminf _{n \rightarrow+\infty} E_{n}\left(\tilde{G}_{n} ; Q^{\eta}\right) \\
& \geq \operatorname{Per}_{1}\left(T_{\tau}(u) ; Q^{\eta}\right)=\operatorname{Per}_{1}\left(T_{\tau}(u) ;(0,1] \times(0, L]\right)
\end{aligned}
$$

as claimed.

Proof of the upper bound. We can use the same recovery sequence as in the proof of the upper bound in Theorem 6. Indeed, the approximation used in the proof of Proposition 5 is compatible with the addition of the boundary term $\phi_{\tau}$, which is continuous along the sequences constructed therein. Moreover, the additional interactions taken into account in $F_{n}^{\#}$ asymptotically give the term with $\phi_{\tau}$.

\subsection{Analysis of minimum problems}

We now briefly describe the behaviour of minimum problems for $F_{\tau}^{\#}$ in dependence of $\tau, \sigma$ and $L$. In order to understand the behaviour of minimizers, it is convenient to refer to the two-dimensional interpretation of the energies, where the effect of the mismatch in periodicity can be seen as the necessity to consider the extension of subsets 
on $(0, L) \times(0,1)$ by periodicity on the Bravais lattice generated by $(L, 1-\tau)$ and $(0,1)$, as in the definition of $T_{\tau}$ above. Note that this extension has no significant energetic effect for sets as the ones in Fig. 7, but it might for sets as in Fig. 6, in particular for rectangles corresponding to constant $u$. This will lead to a more complex typology of minimizers.

We first note that in order to compute minimum values, we can always reduce to piecewise-constant functions, as in the previous analysis of minimum values of $F^{L, \sigma}$. Note moreover that, setting $\tilde{u}(x)=u(L-x)$, we have

$$
F_{\tau}^{\#}(u)=F_{\tau}^{\#}(1-\tilde{u})
$$

Hence, we may limit the study to the case $\sigma \leq \frac{1}{2}$. Recalling that $F_{\tau}(u)=F_{1-\tau}(\tilde{u})$ (see Remark 12) we can also assume $\tau \leq \frac{1}{2}$.

We start by showing that we can assume $u$ monotone non-increasing. Indeed, let $u$ be a piecewise-constant function in $[0, L]$ and denote by $u_{d}$ the non-increasing rearrangement of $u$. Let $z_{m}=\min \{z: u(z)=m=\min u\}$ and $z_{M}=\min \{z: u(z)=$ $M=\max u\}$. If $z_{m} \geq z_{M}$, then

$$
F_{\tau}^{\#}(u)-F_{\tau}^{\#}\left(u_{d}\right) \geq u(L)-u(0)+\phi_{\tau}(u(0), u(L))-\left(m-M+\phi_{\tau}(M, m)\right) .
$$

Since for $\tau \leq \frac{1}{2}$ the function $y-x+\phi_{\tau}(x, y)$ turns out to be non-increasing along any direction $(h,-k)$ with $h, k \geq 0$, we deduce that

$$
u(L)-u(0)+\phi_{\tau}(u(0), u(L)) \geq m-M+\phi_{\tau}(M, m)
$$

showing that $F_{\tau}^{\#}(u) \geq F_{\tau}^{\#}\left(u_{d}\right)$. If $z_{m}<z_{M}$, the conclusion follows by recalling (44) and noting that for $\tau \leq \frac{1}{2}$ the function $x-y+\phi_{\tau}(x, y)$ is non-increasing along any direction $(-h, k)$ with $h, k \geq 0$. Indeed

$$
\begin{aligned}
F_{\tau}^{\#}(u)-F_{\tau}^{\#}\left(u_{d}\right) & \geq u(0)-u(L)+\phi_{\tau}(u(0), u(L))-\left(m-M+\phi_{\tau}(M, m)\right. \\
& \geq u(0)-u(L)+\phi_{\tau}(u(0), u(L))-\left(m-M+\phi_{\tau}(m, M)\right) \geq 0 .
\end{aligned}
$$

Hence, we may assume that $u$ has the form $u(0) \chi_{(0, y)}+u(L) \chi_{(y, L)}$ with $u(0) \geq u(L)$ (and with the integral constraint $u(0) y+u(L)(L-y)=\sigma)$. If $u(0)<1$ and $0<u(L)$, then the monotonicity of $x-y+\phi_{\tau}(x, y)$ ensures that

$$
F_{\tau}^{\#}(u) \geq F_{\tau}^{\#}\left(u_{\sigma}\right)
$$

where $u_{\sigma}$ is the constant function with value $\sigma$. Moreover, $F_{\tau}^{\#}(u)=F_{\tau}^{\#}\left(u_{\sigma}\right)$ if and only if $\tau \leq \sigma$ and $u(0)-u(L) \leq \tau$. Concluding, we again end up with four cases, pictured in Fig. 10. In order to take into account all cases with a common notation, we set

$$
\tau_{*}=\min \{\tau, 1-\tau\}, \quad \tau^{*}=\max \{\tau, 1-\tau\} .
$$




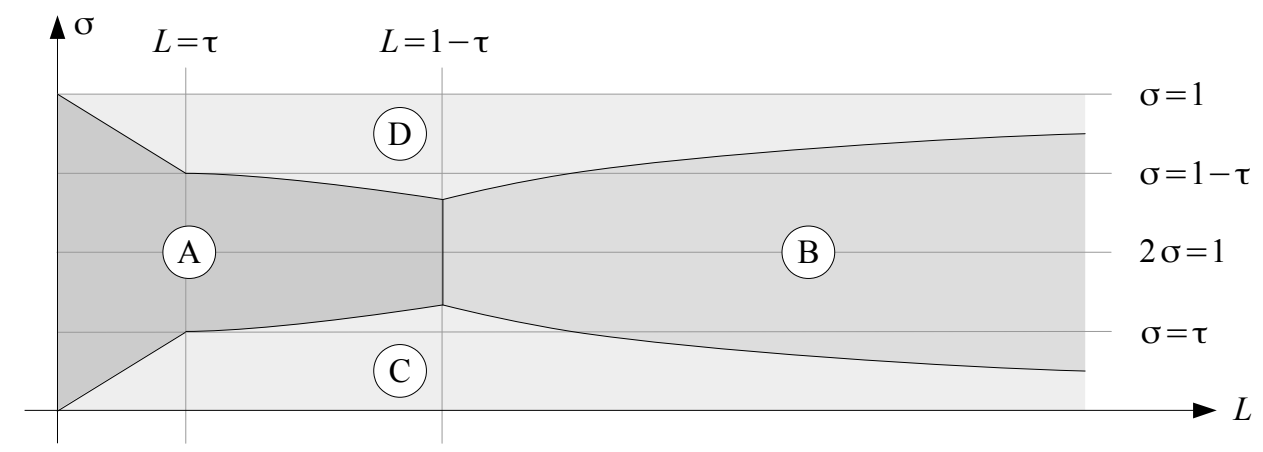

Figure 10: Description of minimum problems at given $\tau \in(0,1 / 2)$

A) If $(L, \sigma)$ satisfies one of the following conditions

$$
\begin{array}{lll}
L \leq \tau_{*} & \text { and } & L \leq \sigma \leq 1-L \\
\tau_{*} \leq L \leq \tau^{*} & \text { and } & \frac{\left(L+\tau_{*}\right)^{2}}{4 L} \leq \sigma \leq 1-\frac{\left(L+\tau_{*}\right)^{2}}{4 L}
\end{array}
$$

then a minimizer is $u_{\min }=\sigma$, with energy $F_{\tau}^{\#}\left(u_{\min }\right)=2 L+2 \min \left\{\sigma, 1-\sigma, \tau_{*}\right\}$. Note that if $\tau=0$ the conditions for $(L, \sigma)$ are described by $L \leq 1$ and $\frac{1}{4 L} \leq \sigma \leq 1-\frac{1}{4 L}$, similarly to the situation pictured in Fig. 5. The minimizer is unique only if $\sigma<\tau_{*}$ or $\sigma>\tau^{*}$ (see Remark 13);

B) if $L \geq \tau^{*}$ and

$$
\frac{1}{4 L} \leq \sigma \leq 1-\frac{1}{4 L}
$$

then a minimizer is the characteristic function $u_{\min }=\chi_{[0, \sigma L]}$. The energy is $F_{\tau}^{\#}\left(u_{\min }\right)=$ 2. Note that all other minimizers are the translations of $u_{\min }$;

C) otherwise, if $\sigma \leq \frac{1}{2}$, the minimum is obtained by minimizing on functions of the form $u=\frac{\sigma L}{y} \chi_{[0, y]}$. In this case, $F_{\tau}^{\#}\left(u_{\min }\right)=4 \sqrt{\sigma L}$, and again all other minimizers are obtained by translation;

D) finally, in the remaining cases, the minimum is obtained by minimizing on functions of the form $u=1-\frac{(1-\sigma) L}{y} \chi_{[0, \sigma y]}$. In this case, $F_{\tau}^{\#}\left(u_{\min }\right)=4 \sqrt{(1-\sigma) L}$, and again all other minimizers are obtained by translation.

Remark 13 (size effect in the periodic case). We limit our description to $\sigma \leq 1 / 2$, and we consider $\tau_{*} \in\left(0, \frac{1}{2}\right)$, analyzing separately the limit cases $\tau_{*}=0$ and $\tau^{*}=\frac{1}{2}$.

Small-size domain: if $L<\tau_{*}$ we have

i) for $\sigma<L$ the minimizers are in the case (C) above

$$
\sqrt{\sigma L} \chi_{[s, \sqrt{\sigma L}+s]} \text { with } s \leq L-\sqrt{\sigma L}
$$


ii) for $\sigma=L$ the expressions in (47) define the constant function $u=L$, which is the only minimizer of the energy. Note that the same holds, if $\sigma \geq \frac{1}{2}$, in the case $\sigma=1-L$

iii) for $L<\sigma<\tau_{*}$ the minimizer is the constant $u=\sigma$;

iv) for $\sigma \geq \tau_{*}$ the minimizers are all monotone non-increasing $u$ satisfying the integral constraint and the boundary conditions $u(0)-u(L) \leq \tau_{*}$.

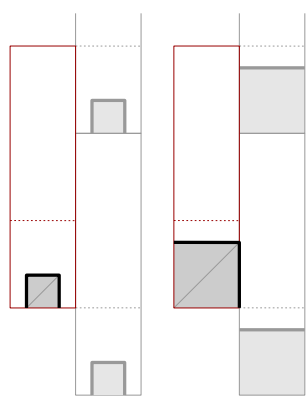

$\sigma<L$

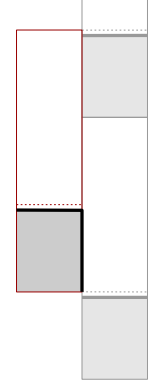

$L<\sigma<\tau$
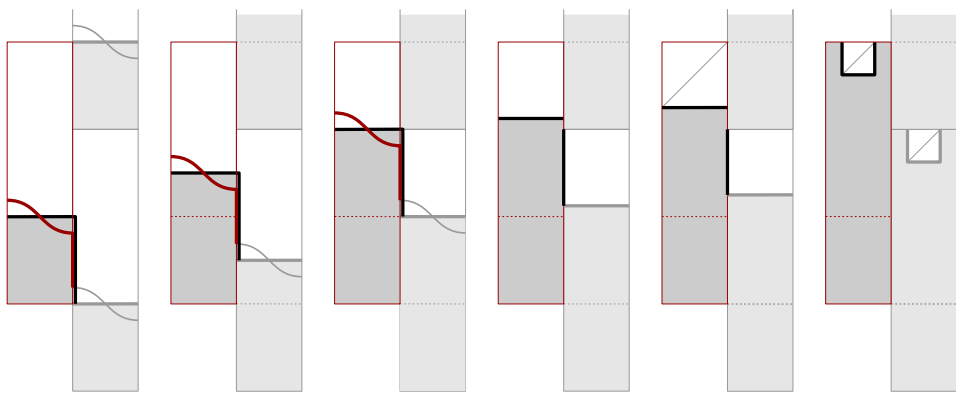

$\sigma=\tau$

$\sigma=1-$

$\sigma=1-L$

$\sigma>1-L$

Figure 11: Minimizers of the energy with varying $\sigma$ for small-size domains

Note that at $\sigma=\tau_{*}$, we have a discontinuity in the form of minimizers. The evolution of the form of the minimizers if $L<\tau_{*}$ is pictured in Fig. 11. For the other cases the form of minimizers can be similarly described and we refer to the figures in Section 4.2 for the necessary changes.

Intermediate-size domain: if $\tau_{*}<L<\tau^{*}$ then we have

i) for $\sigma<\frac{\left(L+\tau_{*}\right)^{2}}{4 L}$ the minimizers are the functions in $(47)$;

ii) for $\sigma=\frac{\left(L+\tau_{*}\right)^{2}}{4 L}$ the minimizers are the functions of the form

$$
\frac{L+\tau_{*}}{2} \chi_{\left[s, \frac{L+\tau_{*}}{2}+s\right]} \text { if } s \leq \frac{L-\tau_{*}}{2}
$$

the constant $u=\sigma$ and all monotone non-increasing $u$ satisfying the integral constraint and the boundary conditions $u(0)-u(L) \leq \tau_{*}$;

iii) for $\frac{\left(L+\tau_{*}\right)^{2}}{4 L}<\sigma$ the minimizers are all monotone non-increasing $u$ satisfying the integral constraint and the boundary conditions $u(0)-u(L) \leq \tau_{*}$.

The evolution of the form of the minimizers is similar to the situation described in Fig. 6 for the non-periodic case, with a different critical threshold: here, the discontinuity appears at $\sigma=\frac{\left(L+\tau_{*}\right)^{2}}{4 L}$ and corresponds to a critical value $\frac{L+\tau_{*}}{2}$.

Large-size domain: if $L>\tau^{*}$ then we have 
i) for $\sigma<\frac{1}{4 L}$ the minimizers are the functions in (47);

ii) for $\sigma=\frac{1}{4 L}$ the minimizers are the functions in (47) which in this case become

$$
\frac{1}{2} \chi_{\left[s, \frac{1}{2}+s\right]} \quad \text { if } \quad s \leq L-\frac{1}{2} \quad \text { and } \quad \frac{1}{2} \chi_{\left[0, \frac{1}{2}-s\right] \cup\left[\frac{1}{2}, L\right]} \quad \text { if } \quad s>L-\frac{1}{2} ;
$$

and the characteristic functions of the form $\chi_{\left[s, s+\frac{1}{4}\right]}$ and $\chi_{\left[0, \frac{1}{4}-s\right] \cup[s, L]}$;

iii) for $\frac{1}{4 L}<\sigma$ the minimizers are the characteristic functions of the form $\chi_{\left[s, s+\frac{1}{4}\right]}$ and $\chi_{\left[0, \frac{1}{4}-s\right] \cup[s, L]}$.

Again, the evolution of the form of the minimizers can be described as in Fig. 7 for the non-periodic case, with a discontinuity at $\sigma=\frac{1}{4 L}$.

Note that if $\tau_{*}=0$ or $\tau_{*}=\frac{1}{2}$, then we only have two regimes (for domains with $L<1$ and with $L>1$ ) as in the non-periodic case.

Remark 14. Note that $0 \in \tau(L)$ for any $L>0$ (see Remark 9), so that for all $\sigma$

$$
\inf _{\tau \in \tau(L)} \min \left\{F_{\tau}^{\#}(u)\right\}=\min \left\{F_{0}^{\#}(u)\right\}
$$

where the minimum is attained in $\tau=0$. Moreover, the minimum value of the functional $F_{\tau}^{\#}$ is independent of $\tau$, in the following cases:

$$
L \geq 1 ; \quad L<1 \text { and } L \leq \min \{\sigma, 1-\sigma\} ; \quad L<1 \text { and } \frac{L}{4} \geq \min \{\sigma, 1-\sigma\},
$$

when it equals the minimum value of the functional $F_{0}^{\#}$.

\section{References}

[1] R. Alicandro, A. Braides, M. Cicalese. Phase and anti-phase boundaries in binary discrete systems: a variational viewpoint. Netw. Heterog. Media 1 (2006), 85-107.

[2] R. Alicandro, M. Cicalese, and A. Gloria. Integral representation of the bulk limit of a general class of energies for bounded and unbounded spin systems. Nonlinearity 21 (2008), 1881-1910.

[3] R. Alicandro and M. S. Gelli. Local and non local continuum limits of Ising type energies for spin systems. SIAM J. Math. Anal. 48 (2016), 895-931.

[4] L. Ambrosio and A. Braides. Functionals defined on partitions of sets of finite perimeter, II: semicontinuity, relaxation and homogenization. J. Math. Pures. Appl. 69 (1990), 307-333. 
[5] A. Braides. Г-convergence for Beginners. Oxford University Press, Oxford, 2002.

[6] A. Braides. An example of non-existence of plane-like minimizers for an almostperiodic Ising system. J. Stat. Phys. 157 (2015), 295-302.

[7] A. Braides, A. Causin and M. Solci. Interfacial energies on quasicrystals. IMA J. Appl. Math. 77 (2012), 816-836.

[8] A. Braides and M. Cicalese. Interfaces, modulated phases and textures in lattice systems. Arch. Ration. Mech. Anal. 223 (2017), 977-1017.

[9] A. Braides and L. Kreutz. Optimal bounds for periodic mixtures of nearestneighbour ferromagnetic interactions. Atti Accad. Naz. Lincei Rend. Lincei Mat. Appl. 28 (2017), 103-117.

[10] A. Braides and L. Kreutz. Optimal design of mixtures of ferromagnetic interactions, arXiv:1610.06455

[11] A. Braides and A. Piatnitski. Homogenization of surface and length energies for spin systems. J. Funct. Anal. 264 (2013), 1296-1328.

[12] A. Braides and M. Solci. Interfacial energies on Penrose lattices. M3AS 21 (2011), $1193-1210$.

[13] A. Braides and L. Truskinovsky. Asymptotic expansions by Gamma-convergence. Cont. Mech. Therm. 20 (2008), 21-62.

[14] L. Caffarelli and R. de la Llave. Interfaces of ground states in Ising models with periodic coefficients. J. Stat. Phys. 118 (2005), 687-719.

[15] L. Lovász. Large Networks and Graph Limits. American Mathematical Society, Providence, 2012. 